\title{
OPTIMAL CONTROL AND SHAPE OPTIMIZATION OF AORTO-CORONARIC BYPASS ANASTOMOSES
}

\author{
ALFIO QUARTERONI ${ }^{1,2, *}$, GIANLUIGI ROZZA ${ }^{1, \dagger}$ \\ ${ }^{1}$ Chair of Modelling and Scientific Computing (CMCS), \\ Institute of Analysis and Scientific Computing (IACS), \\ École Polytechnique Fédérale de Lausanne, \\ CH-1015, Lausanne, Switzerland. \\ ${ }^{2}$ MOX, Dipartimento di Matematica "Francesco Brioschi", \\ Politecnico di Milano, \\ 20133, Milano, Italy.
}

Received 10 July 2003

Revised 4 July 2003

Communicated by N.Bellomo

\begin{abstract}
In this paper we present a new approach in the study of Aorto-Coronaric bypass anastomoses configurations. The theory of optimal control based on adjoint formulation is applied in order to optimize the shape of the zone of the incoming branch of the bypass (the toe) into the coronary. The aim is to provide design indications in the perspective of future development for prosthetic bypasses. With a reduced model based on Stokes equations and a vorticity functional in the down field zone of bypass, a Taylor like patch is found. A feedback procedure with Navier-Stokes fluid model is proposed based on the analysis of wall shear stress and its related indexes such as OSI.

Keywords: Optimal Control; Flow Control; Shape Optimization; Adjoint Problem; Haemodynamics; Aorto-Coronaric Bypass Anastomoses; Oscillatory Shear Index (OSI); Wall Shear Stress; Design of Improved Medical Devices; Finite Elements; Navier Stokes Equations.
\end{abstract}

AMS Subject Classification: 35Q30, 49J20, 65K10, 65N30, 76D55, 76D07, 92C10, 92C50.

\section{Introduction and problem setting}

The surgical realization of a bypass to overcome a critically stenosed artery is a very common practice in everyday cardiovascular clinic (Figure $(1)^{36}$ ).

Fig. 1. Simplified bypass model.

*Alfio.Quarteroni@epfl.ch

†Gianluigi.Rozza@epfl.ch 
Improvement in the understanding of the genesis of coronary diseases is very important as it allows to reduce surgical and post-surgical failures. It may also suggest new means in bypass surgical procedures with less invasive methods and to devise new shape in bypass configuration (see Perktold et al. ${ }^{33}$, O'Brien and McGloughin ${ }^{32}$ ). Generally speaking, mathematical modelling and numerical simulation can allow better understanding of phenomena involved in vascular diseases (see Abdoulaev et al. $^{1}$, Quarteroni, Tuveri and Veneziani ${ }^{35}$, Quarteroni and Formaggia ${ }^{36}$, Fung ${ }^{13}$ ). In this work, the background provided by mathematical modelling and numerical simulation has led us to apply the Optimal Control theory of systems governed by partial differential equations (PDE) with the aim of optimizing the shape of a simplified bypass model.

Optimal control of one (or several) aspect of the problem entails the minimization of a cost functional which describes physical quantities involved in the specific problem. Optimization process is carried out by a control function which, depending upon the context, may represent initial or boundary conditions, shape of the domain (by boundary variations), force terms, sources, etc. In computational fluid dynamics two different kind of optimal control problems can be faced.

The former is called Data Control, in which case the control is on different variables of the problem such as initial and boundary conditions, force terms, sources and coefficients. Control function can be put on the boundary or on a part of it, on the whole computational domain or on a part of it. When control is put on the flux we talk about flow control problem (Becker ${ }^{3}$, Berggren ${ }^{4}$, Hinze and Kunish ${ }^{17}$, Ravindran and $\mathrm{Hou}^{18,19}$, Gunzburger ${ }^{14}$, Slawig $\left.{ }^{39}\right)$.

The latter is called Domain Control, since the solution of the system of PDE is controlled by boundary variations of the domain itself. This field is related with shape optimization and optimal shape design (see the works by A.Jameson et al. ${ }^{20,21,22}$ and Pironneau ${ }^{34}$, Mohammadi and Pironneau ${ }^{29}$, Kawohl et al. ${ }^{23}$, Di Césaré ${ }^{12}$ ), in the context of aerodynamics for external flows (wings and airfoils design) and internal flows (nozzles, channels).

Here we aim at applying the optimal control theory for PDE systems to a specific problem involving both flow control and shape optimization in haemodynamics phenomena. Flow control is involved in the observation of the evolving system and in cost functionals (such as vorticity, wall shear stress), optimal shape design is involved in boundary variations by a control function used as parameter in shaping the bypass graft.

The adjoint approach proposed by J.L. Lions ${ }^{26}$ to get cost functionals gradient in problem with distributed or boundary control and observation has been developed. This approach is computationally cheaper than other approaches using automatic differentiation for the computation of the gradient of the cost functional. In the functional optimization process a descent gradient-based method is used (see Ciarlet $^{6}$ ). Numerical approximation is based on Galerkin-Finite Elements Method ( see Quarteroni and Valli ${ }^{37}$ ). Algorithms were developed for control and optimization as well as for the coupling between state and adjoint problems. 
This problem is suitable to be studied by optimal control theory to let a numerical simulation in moving domains, modified by shape control iterative process to reduce cost functional (in our case, either the down-field vorticity or the wall shear stress). At the end of the study a cuffed bypass is found with a shape which resembles the Taylor arterial patch (Cole, Watterson and O'Reilly ${ }^{7}$ ).

This paper is organized as follows. After this section as introduction and problem setting, in Sec. 2 we deal with clinical aspects of bypass problem (surgery, materials), then in Sec. 3 we introduce numerical (integral) quantities useful to apply optimal control in design processes concerning wall shear stress trend along wall and oscillations in time. In Sec. 4 we deal with a general presentation of optimal control approach, then in Sec. 5 we study the mathematical modelling of the problem (geometrical model, state equations). In Sec. 6 we deal with optimization and control algorithm aspects, then in Sec. 7 we introduce numerical results and a proposed feedback procedure. In the last Sec. 8 we mention some possible future development.

\section{Clinical aspects: surgery, materials, state of the art problems.}

When a coronary artery is affected by a stenosis, the heart muscle can't be properly oxygenated through blood. Aorto-coronaric anastomosis restores the oxygen amount through a bypass surgery downstream an occlusion (see Figure(2)).

At present, different kind and shape for aorto-coronaric bypass anastomoses are available and consequently different surgery procedures are used to set up a bypass. A bypass can be made up either by organic material (e.g. the saphena vein taken

Fig. 2. Heart, coronary arteries and bypass

from patient's legs or the mammary artery) or by prosthetic material. The current saphenous bypass solution requires the extraction of saphena vein with possible complications. In this respect, prosthetic bypasses are less invasive. They may feature very different shape for bypass anastomoses, such as, e.g., cuffed arteriovenous access grafts (Figure (3) and (4)). Different cuffed models are used such as Taylor Patch $^{9}$ and Miller Cuff Bypass ${ }^{11}$, but also standard end-to-side anastomoses at different graft angle ${ }^{10}$ or other shaped carbon-fiber prostheses. For a discussion and a comparative approach see Cole et al. ${ }^{8,7}$.

Fig. 3. Different anastomoses configurations: end-to-side (top) and cuffed on the bed (bottom).

In the cardiovascular system altered flow conditions such as separation, flow reversal, low and oscillatory shear stress areas and abnormal pulse pattern are all recognized as potentially important factors in the development of arterial diseases (White et 
al. ${ }^{44}$, Loth et al. $\left.{ }^{28}\right)$. A detailed understanding of local haemodynamics phenomena and the effect of vascular wall modification on flow patterns can have useful clinical applications especially in surgical procedures or prostheses tailoring (see Steinman et al. ${ }^{40}$, Moore et al. ${ }^{30}$ ). Concerning bypass setting, lots of different aspects and post-surgical complications have to be taken into consideration, among them we mention intimal thickening hyperplasia (near suture lines), restenosis, surgical injury, long term graft failure. Every year $8 \%$ of all patients risk bypass occlusion, after ten years $80 \%$ bypasses must be replaced (Data taken from HeartCenterOnLine). Repeating procedures typically carry a higher risk of complications.

For all these different aspects the design of artificial arterial bypass is a very complex problem. Carbon fiber and Collagen cuffed grafts instead of natural saphenous vein can be used for studying new shape design without needing "in loco" reconstruction. In this framework, Optimal Control by flow control and shape optimization provide a new interesting approach to the problem, with the goal of improving arterial bypass graft on the basis of a better understanding of fluid dynamics aspects involved in the bypass studying.

\section{Design Control Quantities}

In the literature some physical quantities (called indexes) have been proposed in order to measure, at some extent, arteries occlusion risk (or re-occlusion after bypass surgery). These indexes have been introduced in order to enlight some specific mechanism that could be correlated to intimal thickening. A first index stems from the observation that a crucial role is played by blood flow oscillations during the diastolic phase of every single heart beat. Another index attemps at measuring the rapid variations of the shear stress on the vascular wall. In any case these and other indexes (that we are going to review briefly) could be considered as a starting point on the way to synthesize the enormous amount of information given by numerical simulation for the bypass design.

\subsection{Oscillatory Shear Index (OSI)}

The Oscillatory Shear Index (OSI) was introduced in 1983 by Zarins and Giddens ${ }^{46}$ to identify the occlusion risk zones. It is defined as follows:

$$
O S I=\frac{1}{2}\left(1-\frac{\int_{0}^{T} \tau_{w} d t}{\int_{0}^{T}\left|\tau_{w}\right| d t}\right),
$$

where $[0, T)$ is the time interval of a single heart beat $(T \simeq 1 \mathrm{sec})$ and $\tau_{w}$ is the wall shear stress. In a Newtonian fluid model,

$$
\tau_{w}=\mu \frac{\partial \hat{v}}{\partial \hat{n}} \cdot \hat{\tau},
$$


where $\hat{v}$ is the blood velocity field, $\mu$ the blood viscosity, $\hat{n}$ and $\hat{\tau}$ respectively normal and tangential unit vector on the arterial wall. OSI measures the temporal oscillations of the shear stress pointwise, without taking into account the shear stress trend in the immediate neighborhood of a specific (critical) point.

\subsection{Mean Wall Shear Stress Gradient (MWSSG)}

Another indicator of occlusion risk zones is the mean wall shear stress gradient (MWSSG) (see Veneziani ${ }^{42}$ and Wells ${ }^{43}$ ) defined as:

$$
M W S S G=\frac{1}{T} \int_{0}^{T}|W S S G| d t,
$$

where WSSG is the wall shear stress gradient and is defined as follow:

$$
W S S G=\frac{\partial \tau_{w}}{\partial \hat{\tau}}=\nabla \tau_{w} \cdot \hat{\tau} .
$$

According to MWSSG, the zones featuring occlusion risk or abnormal flow pattern are those where strong variations in the shear stress along the wall occur. This index is dual with respect to the OSI, since it weighs spatial variations.

\subsection{Other Indexes}

An alternative to OSI is the Oscillatory Flow Index (OFI), see Taylor et al. ${ }^{41}$ :

$$
O F I=\frac{1}{2}\left(1-\frac{\int_{0}^{T} Q d t}{\int_{0}^{T}|Q| d t}\right),
$$

where $Q$ is the flow rate across an artery section $\Gamma_{f}$,

$$
Q=\int_{\Gamma_{f}} \rho \hat{v} \cdot \hat{n} d \Gamma,
$$

being $\rho$ the blood density. It quantifies temporal oscillations in flow rate.

A problem arising when using one of the previous functionals for optimization purposes is that they don't have a quadratic form. We can use these quantities for feed-back processes, but we need functionals which can provide a good average in time and in space of shear stress trend. In this spirit, we propose other integral quantities that can be defined along the artery wall $\Gamma_{w}$ (or a critical part of it). Set for all $t>0$ :

$$
\Sigma(t)=\int_{\Gamma_{w}}\left(\frac{\partial \tau_{w}(t)}{\partial t}\right)^{2} d \Gamma
$$

and

$$
\mathcal{Q}(t)=\int_{\Gamma_{w}}\left(\frac{\partial Q(t)}{\partial t}\right)^{2} d \Gamma
$$


Then we define:

$$
J_{\tau}=\operatorname{mean}_{[0, T]} \Sigma(t)=\frac{1}{T} \int_{0}^{T} \Sigma(t) d t=\frac{1}{T} \int_{0}^{T} \int_{\Gamma_{w}}\left(\frac{\partial}{\partial t} \tau_{w}(t)\right)^{2} d \Gamma d t
$$

which is the $L^{2}-$ norm of the wall shear stress rate $\left(L^{2}-W S S R\right)$. Similarly, we define the analogous $L^{2}-$ norm of the flow rate $Q\left(L^{2}-F R\right)$ :

$$
J_{Q}=\operatorname{mean}_{[0, T]} \mathcal{Q}(t)=\frac{1}{T} \int_{0}^{T} \mathcal{Q}(t) d t=\frac{1}{T} \int_{0}^{T} \int_{\Gamma_{f}}\left(\frac{\partial}{\partial t} Q\right)^{2} d \Gamma d t
$$

These new indexes $J_{\tau}$ and $J_{Q}$ could be used as cost functionals for the control procedure that we are about to introduce.

\section{A Control approach: looking for cost functional optimization}

In this section we review briefly the optimal control theory based on the adjoint problem. For a general presentation of this subject the interested reader can refer to Lions ${ }^{26,27}$ and Aziz et al. ${ }^{2}$. For functional analysis elements see Yosida ${ }^{45}$, Brezzi and Gilardi ${ }^{5}$.

The mathematical ingredients of an optimal control problem are:

- A Control Function $w$ which belongs to a functional space $\mathcal{U}_{a d}$, called admissible control space.

- A state system $y(w)$ associated to the control $w$, which is the solution of a PDE problem defined on the domain $\Omega$, for instance

$$
\Lambda y(w)=f+B w
$$

where $f$ is a given function, while $\Lambda$ and $B$ are two linear operators. The latter equation is called state equation and represents the physical system to control and optimize. In our case the state equation will be given by either the Stokes or the Navier-Stokes equations. Note $w \rightarrow y(w)$ is an affine map.

- An observation function $z(w)$ belonging to a suitable space $\mathcal{Z}$ which is related to $y(w)$ through an operator $\mathcal{C}, z(w)=\mathcal{C} y(w)$. Our observation will coincide with the restriction of $z(w)$ upon a convenient subset of the computational domain $\Omega$, so in our case $\mathcal{C}$ is indeed a restriction operator.

- A cost functional $J(w)$ (to be minimized) which is a positive quantity depending on the observation $z(w)$. In our problem $J(w)$ could be either $J_{\tau}$ or $J_{Q}$, or another simplified functional depending on the flow vorticity that we will consider in connection with the Stokes equations.

Note that this formulation covers the case where the control variable is a boundary condition or a source term. Optimal shape design is a special case of control theory where the control is the boundary itself $(w \rightarrow \Omega(w) \rightarrow y(w)$, where $w \rightarrow \Omega(w)$ is the parameterization of the domain by a control parameter $w$ and the problem is to minimize a functional $J(w, y(w))$, which depends on $w$ through $y(w)$ but also 
directly (for instance $J$ is an integral of a quantity depending on $y(w)$ extended to a region depending on $w)$ ). (See Mohammadi and Pironneau ${ }^{29}$ ).

In our problem the control will be a subset of the domain boundary $\Gamma(w)$. The general formulation of an optimal control problem reads:

- Find a function $w \in \mathcal{U}_{a d}$ so that:

$$
J(w)=\inf \left\{J(v), \quad \forall v \in \mathcal{U}_{a d}\right\} .
$$

With the sole sake of clarity for the reader unfamiliar with control theory, a classical example follows. We assume that $\mathcal{U}$ is a Hilbert space, $\pi$ a bilinear continuous form on $\mathcal{U}$, coercive and symmetric, $L$ a linear continuous functional on $\mathcal{U}$ and $\mathcal{U}_{a d} \subset \mathcal{U}$ is the admissible control functions space. If we assume that the cost functional is given by:

$$
J(v)=\pi(v, v)-2 L(v), \forall v \in \mathcal{U}_{a d},
$$

under the previous hypotheses on $\pi$, there exists a unique control $w \in \mathcal{U}_{a d}$. Moreover, (4.12) can be replaced by an equivalent variational inequality:

$$
J^{\prime}(w) \cdot(v-w) \geq 0 \quad \forall v \in \mathcal{U}_{a d},
$$

under the hypothesis that the function $v \rightarrow J(v)$ is strictly convex, differentiable and satisfies: $J(v) \rightarrow+\infty$ as $\|v\| \rightarrow+\infty$. The last hypothesis may be omitted if $\mathcal{U}_{a d}$ is bounded. For example assume that the cost functional is given by:

$$
J(v)=\left\|\mathcal{C} y(v)-z_{d}\right\|_{\mathcal{Z}}^{2},
$$

where $z_{d}$ is a given optimal condition (to be reached) in the observation space $\mathcal{Z}$. Noting that:

$$
J(v)=\left\|\mathcal{C}[y(v)-y(0)]+\mathcal{C} y(0)-z_{d}\right\|_{\mathcal{Z}}^{2},
$$

and defining:

$$
\begin{gathered}
\widetilde{\pi}(w, v)=(\mathcal{C}[y(w)-y(0)], \mathcal{C}[y(v)-y(0)]) \mathcal{Z}, \\
L(v)=\left(z_{d}-\mathcal{C} y(0), \mathcal{C}[y(v)-y(0)]\right) \mathcal{Z},
\end{gathered}
$$

we can reformulate our cost functional as follows:

$$
J(v)=\widetilde{\pi}(v, v)-2 L(v)+\left\|z_{d}-\mathcal{C} y(0)\right\|_{\mathcal{Z}}^{2} .
$$

The inequality (4.14) is equivalent to:

$$
\left(\mathcal{C} y(w)-z_{d}, \mathcal{C}[y(v)-y(w)]\right)_{\mathcal{Z}} \geq 0, \forall v \in \mathcal{U}_{a d} .
$$

Introducing the adjoint operator $\mathcal{C}^{*}$ of $\mathcal{C}$ and a Hilbert space $\mathcal{V}$ of test functions, so that:

$$
\mathcal{V}^{\prime}<\mathcal{C}^{*} z, v>_{\mathcal{V}}={ }_{\mathcal{Z}^{\prime}}<z, \mathcal{C} v>_{\mathcal{Z}}, \forall v \in \mathcal{V}, z \in \mathcal{Z}^{\prime}
$$


expression (4.16) becomes:

$$
\left(\mathcal{C}^{*}\left(\mathcal{C} y(w)-z_{d}\right), y(v)-y(w)\right)_{\mathcal{Z}} \geq 0, \quad \forall v \in \mathcal{U}_{a d} .
$$

Let $\Lambda^{*}$ denote the adjoint operator of $\Lambda$, that is $\left.\langle\Lambda v, u\rangle=<v, \Lambda^{*} u\right\rangle$. Then for every control $w \in \mathcal{U}$ let us introduce the adjoint state $p(w) \in \mathcal{V}$ which is the solution of the adjoint problem:

$$
\Lambda^{*} p(w)=\mathcal{C}^{*}\left[\mathcal{C} y(w)-z_{d}\right]
$$

After some transformations inequality (4.17) can be written as:

$$
\left(B^{*} p(w), v-w\right)_{\mathcal{U}} \geq 0, \quad \forall v \in \mathcal{U}_{a d},
$$

where $B^{*}$ is the adjoint of the operator $B$. A useful identity relating the gradient of cost functional to the solution of the adjoint problem is:

$$
\frac{1}{2} J^{\prime}(v)=B^{*} p(v)
$$

Thus the evaluation of the gradient of the cost function can be achieved at the expense of solving an additional PDE problem, the so-called adjoint state equation. For this derivation see Lions $^{26}$.

\section{Mathematical modelling of the problem.}

Blood is a very complex fluid which interacts with the compliant arterial vessels. Some simplificatory assumptions are in order in view of applying a control procedure. Concerning the fluid model we consider blood as a Newtonian fluid, modelled by Navier-Stokes equations (or even by the simplified Stokes model). This model is a valid approximation when studying blood flow in large or medium-size arteries. The Reynolds number is rather low, so the flow can be regarded as laminar.

\subsection{The geometrical model}

To model the incoming branch of a bypass, different bypass anastomoses models have been taken into consideration. We have simplified our model by considering a longitudinal section in the mean plane reducing it to a two-dimensional problem. As initial configuration we have used a fiberglass model provided by the Surgical Vascular Division of Aahrus University Hospital (Denmark). To define anastomoses geometry for numerical simulation, different aspects have to be taken into account:

- Fluid dynamics phenomena in the bypass are strictly related with the shape assumed, such as the arising of blood re-circulating zones. Blood pulsatility due to heart beat influences our analysis. The most critical zone in the bypass design is the toe and must be carefully modelled.

- Another important aspect to keep into consideration is the graft angle between the incoming branch and the occluded artery. 
- The diameter of the occluded branch (below) is $D=3.5 \mathrm{~mm}$ and the one of the bypass (above) is $0.96 \cdot D$, as suggested in Quarteroni and Formaggia ${ }^{36}$.

A curvature in the vascular wall is introduced to model coronary arteries in order to take into consideration the presence of the heart muscle. Figure (5) shows the mean plane before applying the shape optimization process.

\subsection{The State problem: Navier-Stokes and Stokes equations}

In our optimal control process we use as state equations the steady Stokes equations which are however well suited for modeling newtonian flows at low Reynolds number. In reality, because of flow pulsatility we should consider the unsteady Navier-Stokes equations. Unfortunately, such a complete fluid model would be too complex to control because of evolution terms, non linearity in the adjoint problem and the fact that the shape of our computational domain depends on time. Nonetheless, after performing shape optimization on our reduced model governed by steady Stokes equations, we will turn our attention to the complete NS model for feed-back procedures considering design cost functionals such as the ones presented in Section 3. In this Section we focus therefore on the steady Stokes problem.

Numerical approximation is based on the Galerkin-finite element method using piecewise linear elements for velocity and pressure fields and SUPG stabilization (see, e.g., Quarteroni and Valli ${ }^{37}$ ). The Stokes equations in a two-dimensional computational domain $\Omega$ with velocity vector $\hat{v}=\{u, v\}$ and pressure $p$ read:

$$
\left\{\begin{array}{l}
-\nu \Delta \hat{v}+\nabla p=0 \text { in } \Omega \subset \mathbb{R}^{2} \\
\nabla \cdot \hat{v}=0 \text { in } \Omega \\
\hat{v}=0 \text { on } \Gamma_{w} \\
\hat{v} \cdot \hat{t}=0, \hat{v} \cdot \hat{n}=g_{\text {in }} \text { on }\left.\Gamma\right|_{\text {in }}, \\
\hat{T} \cdot \hat{n}=0 \text { on }\left.\Gamma\right|_{\text {out }},
\end{array}\right.
$$

where $\hat{n}$ and $\hat{t}$ are, respectively, normal and tangential unit vector on the domain boundary $\partial \Omega$. The latter is partitioned in three components: $\Gamma_{i n}$ is the inflow boundary, $\Gamma_{\text {out }}$ the outflow boundary and $\Gamma_{w}$ the boundary corresponding to the arterial wall; Figure (5) represents schematically the computational geometry and the symbols used.

Fig. 5. Anastomosis schematic model and symbolic notation used for domain and boundary, with observation down-field zone $\Omega_{w d}$.

Concerning boundary conditions, no-slip conditions are imposed on all vascular walls of $\Gamma_{w}$, over the stenosed artery portion and on the incoming branch of bypass. 
At inflow section $\Gamma_{i n}$, a Hagen-Poiseille's velocity profile $g_{i n}$ is imposed, while on outflow section $\Gamma_{\text {out }}$ of the artery free-stress, Neumann-type condition hold:

$$
\hat{T} \cdot \hat{n}=-p \cdot \hat{n}+2 \nu\left(\frac{\nabla \hat{v}+\nabla \hat{v}^{T}}{2}\right) \cdot \hat{n}=0 .
$$

Velocity values at the inflow are chosen in such a way that the Reynolds number $R e=\frac{\tilde{v} \cdot D}{\nu}$ has order $10^{3}$. Blood kinematic viscosity $\nu=\frac{\mu}{\rho}$ is to $4 \cdot 10^{-6} \mathrm{~m}^{2} \mathrm{~s}^{-1}$, blood density $\rho=1 \mathrm{~g} \mathrm{~cm}^{-3}$ and dynamic viscosity $\mu=4 \cdot 10^{-2} \mathrm{~g} \mathrm{~cm}^{-1} \mathrm{~s}^{-1} ; \widetilde{v}$ is a mean inflow velocity related with $g_{i n}$, while $D$ is the arterial diameter $(3.5 \mathrm{~mm})$. Arterial thickness is about $0.5 \mathrm{~mm}^{36}$.

In our problem the control $w$ represents the shape $\Gamma_{w}$ itself or a part $\Gamma_{c}$ of it (typically the incoming branch). Precisely, we write (see Figure (5)):

$$
\Gamma_{w}=\Gamma_{w(\text { rigid })} \cup \Gamma_{c(\text { toe })},
$$

where

$$
\Gamma_{w(\text { rigid })}=\Gamma_{w(\text { bed })} \cup \Gamma_{w(\text { heel })} \cup \Gamma_{w(\text { graft })} \cup \Gamma_{w(\text { stenosis })},
$$

then we can build our geometrical model for $\Gamma_{c}$ (double-dotted in Figure (5)) made up of $M$ branches $\Gamma_{c}^{j}(w)$ represented by:

$$
\Gamma_{c}=\sum_{j=0}^{M} \Gamma_{c}^{j}(w), \Gamma_{c}^{j}(w)=\overline{\Gamma_{c}^{j}}+w^{j},
$$

where $w^{j}$ is the control variable. More precisely we take

$$
\overline{\Gamma_{c}^{j}}(s)=\sum_{i=0}^{N} \alpha_{i j} f_{i j}(s)
$$

where $s$ is the horizontal abscissa that parametrizes the curve $\Gamma_{c}, f_{i j}(s)=$ $\sum_{k=0}^{i} a_{k} s^{k}$ are given shape polynomial functions of $i^{t h}$ order and $\alpha_{i j}$ are suitable weights. The control shape function $w^{j}$ changes along the optimization process. At the $k-t h$ iteration we have:

$$
w_{k}^{j}(s)=\sum_{m=0}^{k-1}\left(\delta w_{m}^{j}\right)(s) .
$$

\subsection{The observation on the system}

After having described the control strategy we have to choose a cost functional. We consider a distributed observation in the down-field zone of the incoming branch of the bypass indicated as $\Omega_{w d}$ (see Figure (5)). For our preliminary study our observation will be the vorticity $\nabla \times \hat{v}=\frac{\partial v}{\partial x}-\frac{\partial u}{\partial y}$; $\hat{v}$ is the solution of the Stokes equations (5.21) and we control the system by minimizing the following functional:

$$
J(w)=\int_{\Omega_{w d}}|\nabla \times \hat{v}|^{2} d \Omega .
$$


By this observation we can draw information about the vorticity distribution. The curl of the vorticity will provide a source term of the adjoint problem, whose solution will be used during our optimization procedure to produce an estimate of the gradient of the cost functional, as we have already pointed in Sec. 4. We underline that we consider not only what happens on the vascular wall but also in the fluid domain. For this reason this is also a flow control problem and not only a shape optimization problem, because an optimal shape design problem is usually based only on the boundary observation. For further information about vorticity reduction problem see Berggren ${ }^{4}$. In this approach we have also taken into consideration another important aspect, concerning wall stiffness and elasticity. Indeed, instead of (5.28) we employ a more complete functional which includes a new term that minimizes the wall deformation in the zone where the bypass merges with the artery:

$$
J(w)=\int_{\Omega_{w d}}|\nabla \times \hat{v}|^{2} d \Omega+\frac{E_{0}}{\xi} \sum_{j}\left(\int_{\Gamma_{c}^{j}(w)} d \Gamma-\widetilde{l}_{j}\right)^{2} .
$$

Here $\tilde{l}_{j}$ is the original length of vascular wall $\Gamma_{c}^{j}(w), \xi$ is a suitable weight, while $E_{0}$ is Young's elasticity modulus $\left(3 \cdot 10^{6}\right.$ dyne $\left.\mathrm{cm}^{-2}\right)$. We are thus considering a multidisciplinary optimization of a prosthetic device. The last term in (5.29) describes the elastic wall behavior and provides a measure for elastic energy and wall deformation. The two quantities on the right end side of (5.29) have to be weighed by the choice of $\xi$.

\subsection{The adjoint problem}

As already mentioned during the optimization process we must solve another PDE problem, the so-called adjoint problem. When considering the cost-functional (5.28), the adjoint problem reads:

$$
\left\{\begin{array}{l}
-\nu \Delta \mathbf{p}+\nabla \sigma=\nabla \times \nabla \times\left.\hat{v}\right|_{\Omega_{w d}} \text { in } \Omega, \\
\nabla \cdot \mathbf{p}=0 \text { in } \Omega \\
\mathbf{p}=0 \text { on } \Gamma_{\text {in }} \cup \Gamma_{\text {out }} \cup \Gamma_{w},
\end{array}\right.
$$

where $\mathbf{p}$ and $\sigma$ denote the adjoint velocity and pressure, respectively.

See for details Berggren ${ }^{4}$, Mohammadi and Pironneau ${ }^{29}$, where a similar problem is studied with the aim of reducing viscous dissipation. No slip condition is imposed not only on the vascular wall $\Gamma_{w}(w)$ but also on $\Gamma_{\text {in }}$ and $\Gamma_{\text {out }}$. An important role is played by the adjoint pressure $\sigma$, which is used to provide indications on the shape modification of $\Gamma_{w}^{j}$. Precisely, at $k-t h$ iteration, we have

$$
\left(\delta w_{k}^{j}\right)(s)=-\left.\xi_{j}(s) \lambda_{j} \sigma_{k}\right|_{\Gamma_{c_{k}}^{j}}
$$

where $\lambda_{j}$ is a relaxation parameter for descent gradient-based method (step-size) (see Quarteroni, Sacco and Saleri ${ }^{38}$ ), while $\xi_{j}(s)$ is a weight for the shape variation, used to satisfy congruence geometrical conditions such as shape continuity and 
guarantee that the internal arterial diameter cannot go below a certain threshold. During the shape optimization process we have two degrees of freedom available $(x, y)$ in the mean plane of the bypass. In fact we use only $y$, constraining $x$ and letting point-wise boundary variations in vertical direction. For this reason the weight parameter $\xi_{j}(s)$ is a function of linear abscissa only.

In the adjoint problem (5.30) the observation is only an internal source in the downfield zone, having operated a restriction on the right hand side.

When we consider the more complete cost functional (5.29), which accounts for wall stiffness, the adjoint problem modifies as follows:

$$
\left\{\begin{array}{l}
-\nu \Delta \mathbf{p}+\nabla \sigma=\nabla \times \nabla \times\left.\hat{v}\right|_{\Omega_{w d}} \text { in } \Omega, \\
\nabla \cdot \mathbf{p}=0 \text { in } \Omega, \\
\mathbf{p}=0 \text { on } \Gamma_{\text {in }} \cup \Gamma_{\text {out }} \cup \Gamma_{w(\text { rigid })}, \\
\mathbf{p} \cdot \hat{n}=\left(\int_{\Gamma_{c}^{j}(w)} d \Gamma-\widetilde{l}_{j}\right) \quad \text { on } \Gamma_{c}^{j}(w) .
\end{array}\right.
$$

\section{Optimization and control algorithm aspects}

A general structure for control algorithm can be described as follows at each iteration step:

- Solve the state problem (5.21) with velocity components $u_{k}$ and $v_{k}$ in the domain $\Omega_{k}$ with the moving boundary $\Gamma_{c_{k}}^{j}\left(w_{k-1}\right)$ obtained from the previous iteration of the control cycle.

- Compute the vorticity $\nabla \times \hat{v}_{k}$ in the domain $\left(\Omega_{w d}\right)_{k}$.

- Evaluation of the cost functional (5.28) or (5.29).

- Solve the adjoint problems (5.30) or (5.32) for $\mathbf{p}_{k}$ and $\sigma_{k}$ with source term $\left(\nabla \times \nabla \hat{v}_{k}\right)$. Also the adjoint problem is numerically stabilized with the same method used for the state problem (5.21).

- Use the stopping test on the adjoint state $p_{k_{i}}(w)$ :

$$
\int_{\Omega_{w d_{k}}}\left(\sum_{i=1}^{2} \mu_{i}^{2} p_{k_{i}}^{2}+\gamma^{2} \sigma_{k}^{2}\right) d \Omega \leq \beta,
$$

for a suitable tolerance $\beta$. This quantity provides a good indication on the cost functional gradient.

- Estimate the shape variation $\delta w_{k}^{j}$ on the boundary $\Gamma_{c_{k}}^{j}\left(w_{k}^{j}\right)$ by a descent gradient-type method (see ${ }^{23}$ ) and by results provided by (4.20):

$$
\left.\delta w_{k}^{j}\right|_{\Gamma_{c_{k}}^{j}}=-\hat{\lambda_{k}} J_{k}^{\prime}\left(w_{k}^{j}\right)=-\left.\xi(s)_{j} \lambda_{k} \sigma_{k}\right|_{\Gamma_{c_{k}}^{j}}
$$

with $0<\lambda_{k} \ll 1$, which causes a vertical shape displacement $\delta w_{k}^{j}$ in (5.25). In our case $\delta w_{k}^{j}$ is modified only by the adjoint pressure $\sigma_{k}$; since no slip boundary conditions are imposed for the adjoint velocity $\mathbf{p}$ on the arterial wall $\Gamma_{c_{k}}$. 
- Update the boundary shape:

$$
\Gamma_{c_{k+1}}^{j}\left(w_{k}^{j}\right)=\overline{\Gamma_{c}^{j}}+w_{k}^{j}+\delta w_{k}^{j}=\overline{\Gamma_{c}^{j}}+\sum_{m=0}^{k}\left(\delta w_{m}^{j}\right) .
$$

- Modify the Boundary and re-construct or adapt the grid (see Section (6.1)).

Fig. 6. Optimal shape design and flow control scheme.

For more on optimal shape design techniques by boundary variations and shape parameters see e.g. Di Césaré ${ }^{12}$. Figure (6) shows a general scheme for a flow control and shape optimization problem.

\subsection{Mesh strategy}

During the shape optimization process the mesh is subject to be modified by boundary variations. There are two different strategies for mesh treatment at every step:

- Grid reconstruction. This process is computationally expensive, however new elements can be added.

- Mesh stretching and elements adaptation after boundary variations operated by small deformation. This approach is faster than the previous one and cheaper. Mesh is usually stretched only in the computational domain surrounding the boundary $\Gamma_{c}$ subject to shape changes.

Our approach used both strategies: the mesh was completely re-built after few iterations, while at each step mesh was stretched by locally boundary variations. In this process conditions were put as control on geometrical quantities of mesh elements such as minimum angles or maximum side-length. 


\section{Numerical Results}

Numerical tests and simulations have been carried out using $\mathrm{Bamg}^{16}$, a Bidimensional Anisotropic Mesh Generator and $\mathrm{FreeFem}^{15}$, a finite element Library developed at INRIA, the French National Institute for Research in Computer Science and Control, with the development of algorithms based on control theory and shape optimization. In this section we are going to present numerical results we got to obtain vorticity reduction and a feedback procedure to prevent wall shear stress oscillations with the functional (3.9). Several remarks are in order:

- Wall curvature (to model arteries near the heart muscle) increases vorticity.

- Graft angle of the bypass incoming branch influences vorticity, by reducing the angle also vorticity is reduced in the down-field observation zone.

- Bypass surgery causes an increase of vorticity in the downfield area of about $35 \%$ than the previous configuration without bypass bridge.

- After obtaining the new optimal shape for the incoming branch in 25 iterations, vorticity reduction by optimal control is about of $45 \%$,

Figures (7)-(11) provide an account of numerical results and show the optimal shape of the bypass obtained using steady Stokes equations in an optimal control problem with cost functionals (5.28) and (5.29). As we can see the shape that is found resembles the Taylor patch configuration ${ }^{7}$, a little cuffed shape. The effect of the Taylor patch is to reduce gradually the average velocity of the blood as it approaches the distal anastomosis, since the cross-sectional area of the bypass is steadily becoming larger. This prevents the sudden deceleration experienced in the conventional model with the fluid returning to the host vessel. There is a gradual reduction in the momentum of the blood while approaching the junction, in fact, the blood is guided more smoothly through the vessel thanks to the gradual changing geometry. Flow disturbances are abated, undesirable flow separation at the toe of the bypass diminished.

Fig. 7. Bypass configuration before optimal shape design process: iso-velocity $\left[\mathrm{cms}^{-1}\right]$.

Fig. 8. Bypass configuration at the end of shape optimization: iso-velocity.

Fig. 9. Bypass configuration near the incoming branch before shape optimization (left) and with $11 \%$ vorticity reduction (right). 
July 4, 2003 11:5 WSPC/INSTRUCTION FILE QR2003-M3AS

Optimal Control and Shape Optimization of Aorto-Coronaric Bypass Anastomoses

Fig. 10. Bypass configuration near the incoming branch with $22 \%$ (left) and with $32 \%$ vorticity reduction (right). 
Fig. 11. Bypass configuration near the incoming branch with $38 \%$ (left) and with $45 \%$ vorticity reduction (right).

\subsection{Feedback procedures}

A feedback procedure has been implemented by solving the unsteady Navier-Stokes equations in the original configuration as well as in the final configuration obtained after applying the optimal shape design process on a reduced model, based on steady Stokes equations. The full Navier-Stokes problem reads, for all $0<t<T$ :

$$
\left\{\begin{array}{l}
\frac{\partial \hat{v}}{\partial t}-\nu \Delta \hat{v}+\hat{v} \cdot \nabla \hat{v}+\nabla p=0 \text { in } \Omega \\
\nabla \cdot \hat{v}=0 \text { in } \Omega \\
\hat{v}=0 \text { on } \Gamma_{w} \\
\hat{v} \cdot \hat{t}=0, \hat{v} \cdot \hat{n}=g_{\text {in }}(t) \text { on }\left.\Gamma\right|_{\text {in }} \\
\hat{T} \cdot \hat{n}=0 \text { on }\left.\Gamma\right|_{\text {out }},
\end{array}\right.
$$

We used Hagen-Poiselle inflow pulsatory condition (with period $T=1 s$ ) and time step $\Delta t=0.01 \mathrm{~s}$. Figures (12)-(13) show unsteady flows at different time steps. During the feedback procedure we used the functional (3.9) to provide useful information about unsteady fluid dynamics phenomena such as wall shear stress oscillations during pulsatory systolic and diastolic phases. This quadratic functional is complete because it keeps into consideration WSS variations in time and the functional is not pointwise but defined on $\Gamma_{c}$, the vascular wall we are modelling by optimal shape design. Shape optimization process guarantees a reduction of $45 \%$ of vorticity using steady Stokes flow model and a reduction of $25 \%$ in wall shear stress oscillations in time (on the vascular wall $\Gamma_{c}$ we are modelling). In general, the flow at the distal junction exhibits considerable spatial and temporal variations. With the adaptation operated by shape optimization technique we find, as already seen, a graft which resembles the Taylor patch configuration in which the anastomotic flow is less disturbed, a less adverse shear stress distribution prevails and furthermore flow separation is reduced ${ }^{7}$. Intimal thickening hyperplasia should be alleviated at the toe in the new bypass configuration proposed. In Figure (14) an

Fig. 12. Fluid dynamics unsteady phenomena into the incoming branch of bypass at $t=0.3 \mathrm{~s}$, velocity $\left[\mathrm{cms}^{-1}\right]$.

Fig. 13. Fluid dynamics unsteady phenomena into the incoming branch of bypass at $t=0.5 s$. 
integral quantity of interest is plotted, it's the wall shear stress variation in time $\Sigma(t)(3.7)$ on $\Gamma_{c}$, (see (3.7)), its calculation has been done to get information about functional $J_{\tau}$ (3.9) which provides integral information in time $(0, T)$ for $\Sigma(t)$. As we can see the oscillatory behaviour of $\Sigma(t)$ on $\Gamma_{c}$ is strongly reduced after applying shape optimization.

Fig. 14. Variations in time of the Wall Shear Stress $\Sigma(t)\left[\mathrm{Nm}^{-1} \mathrm{~s}^{-1}\right]$ (3.7) along $\Gamma_{c}$ (pulsatory inflow condition) in the two different bypass configurations, at the beginning (continuous line) and at the end (dashed line) of optimal shape design process (OSD).

\section{Future developments}

The development of tools for geometry reconstruction from medical data (medical imaging and other non-invasive means) and their integration with numerical simulation could provide improvements in disease diagnosis procedures.

Optimal control and shape optimization applied to fully unsteady incompressible Navier-Stokes equations (see Laporte ${ }^{24}$ and Tallec ${ }^{25}$ ) and possibly the coupled fluidstructure problem (Moubachir and Zolésio ${ }^{31}$ ) and the setting of the problem in a three-dimensional geometry will provide more realistic design indications concerning surgical prosthesis realizations.

A further development will be devoted to build efficient schemes for reduced-basis methodology approximations which could be more efficient for use in a repetitive design environment as optimal shape design methodology requires.

\section{Acknowledgements}

G.Rozza acknowledges the financial support provided through the European Community's Human Potential Programme under contract HPRN-CT-2002-00270 HaeMOdel. This work has also been supported in part by the Swiss National Science Foundation (Project n. 20-65110.01) and by Italian Cofin2001 MURST Project "Metodi numerici avanzati per equazioni alle derivate parziali di interesse applicativo".

\section{References}

1. G. Abdoulaev, S. Cadeddu, G. Delussu, M. Donizzelli, C. Manzi, L. Formaggia, A. Giachetti, E. Gobbetti, A. Leone, P. Pili, A. Schenine, M. Tuveri, A. Varone, A. Veneziani, G. Zanetti, A. Zorcolo. VIVA: the Virtual Vascular Project. IEEE Transactions on Information Technology in Medicine, n 22(4), 1998, pp. 268-274.

2. A.K. Aziz, J.W. Wingate, M.J. Balas. Control Theory of Systems Governed by Partial Differential Equations. Academic Press, 1971.

3. R. Becker. Mesh Adaptation for Stationary Flow Control. Journal of Mathematical Fluid Mechanics, Vol. 3 (2001), pp. 317-341, Birkhäuser Verlag, Basel. 
4. M. Berggren. Numerical Solution of a Flow-Control problem: Vorticity Reduction by Dynamic Boundary Action. SIAM, Journal of Scientific Computing, Vol. 19, No. 3 (1998), pp.829-860.

5. F. Brezzi, G. Gilardi. Functional Analysis and Functional Spaces. Mc-Graw Hill, NY, 1987.

6. P.G. Ciarlet. Introduction à l'Analyse Numérique Matricielle et à l'Optimisation. Dunod, Paris, 1998.

7. J.S. Cole, J.K. Watterson, M.J.G. O'Reilly. Numerical investigation of the haemodynamics at a patched arterial bypass anastomosis. Medical Engineering and Physics, Vol. 24, 2002, pp. 393-401.

8. J.S. Cole, J.K. Watterson, M.J.G. O'Reilly. Is there a haemodynamic advantage associated with cuffed arterial anastomoses. Journal of Biomechanics, Vol. 35, 2002, pp. 1337-46.

9. J.S. Cole, J.K. Watterson, S. Raghunathan and M.J.G. O'Reilly. Numerical Study of Blood Flow Through the Taylor Arterial Bypass Model. $12^{\text {th }}$ Meeting of the European Society of Biomechanics, Dublin 2000.

10. J.S. Cole, J.K. Watterson and M.J.G. O'Reilly. Haemodynamics of Modified Distal Bypass Graft Anastomoses. $7^{\text {th }}$ Annual Conference of the Section of Bioengineering of the Royal Academy of Medicine in Ireland, Arklow 2001.

11. J.S. Cole, L.D. Wijesinghe, J.K. Watterson and D.J.A. Scott. Computational and Experimental Simulations of the Haemodynamics at Cuffed Arterial Bypass Graft Anastomoses. Proceedings of the Institution of Mechanical Engineers, Part H: Journal of Engineering in Medicine, Vol. 216, 2002, pp. 135-143.

12. N. Di Césaré. Outils pour l'Optimisation de forme et le Contrôle Optimal, application à la Mécanique des Fluides. Thèse de Doctorat de l'Université Paris VI, 2000.

13. Y.C. Fung. Biodynamics: Circulation. Springer and Verlag, New York, 1984.

14. M. Gunzburger, L. Hou, T. Svobodny. Boundary Velocity Control of Incompressible Flow with an application to viscous drag reduction. SIAM, Journal of Control and Optimization, Vol. 30, No. 1 (1992), pp. 167-181.

15. F. Hecht, O. Pironneau. Multiple Unstructured Meshes and the Design of Freefem+. INRIA, Rocquencourt, 1999.

16. F. Hecht. BAMG: Bidimensional Anisotropic Mesh Generator. User Guide. INRIA, Rocquencourt, 1998.

17. M. Hinze, K. Kunisch. Second Order Methods for Optimal Control of time-dependent Fluid-Flow. SIAM, Journal of Control Optimization, Vol. 40, No. 3 (2001), pp. 925946.

18. L. Hou, S. Ravindran. Numerical Approximation of Optimal Flow Control Problems by a penalty method: error estimates and numerical results. SIAM, Journal of Scientific Computing, Vol. 20, No. 5 (1999), pp.1753-1777.

19. L. Hou, S. Ravindran. A penalized Neumann Control Approach for solving an Optimal Control Problem for Navier-Stokes equation. SIAM, Journal of Control and Optimization, Vol. 36, No. 5 (1998), pp. 1795-1814.

20. A. Jameson, J.C. Vassberg. Computational Fluid Dynamics for Aerodynamic Design: its current and future impact. AIAA Paper 2001-0538, $39^{\text {th }}$ AIAA Aerospace Sciences Meeting and Exibit, Jan 8-11 2001, Reno NV.

21. A. Jameson. Optimum Aerodynamic Design using CFD and Control Theory. AIAA Paper 95-1729, $12^{\text {th }}$ AIAA Computational Fluid Dynamics Conference 1995.

22. A. Jameson. Aerodynamic Design via Control Theory. Journal of Scientific Computing, Vol. 3 (1988), p. 233-260.

23. B. Kawohl, O. Pironneau, L. Tartar, J.-P. Zolésio.Optimal Shape Design.Springer, 
Berlin, 2000.

24. E. Laporte. Optimisation de formes pour Écoulements Instationnaires. Ph.D Thesis, École Polytechnique, Paris, 1998.

25. E. Laporte, P. Le Tallec. Shape optimization in unsteady flows. INRIA, Rapport de Recherche, No 3693, 1999.

26. J.L. Lions. Optimal Control of Systems Governed by Partial Differential Equations. Springer-Verlag, 1971.

27. J.L. Lions. Some Aspects of the Optimal Control of distribuited parameter Systems. SIAM, Philadelphia, 1972.

28. F. Loth, S.A Jones, D.P. Giddens, H.S Bassiouny, C.K Zarins, S. Glagov. Measurement of Velocity and Wall Shear Stress inside a PTE vascular graft model under steady flow conditions. Journal of Biomechanical Engineering, Vol. 119, pp. 187-194, May 1997.

29. B. Mohammadi, O. Pironneau. Applied Shape Optimization for Fluids. Oxford University Press, Oxford, 2001.

30. J.A. Moore, D.A. Steinman, S. Prakash, C.R. Ethier, K.W Johnston. A numerical study of blood flow patterns in anatomically realistic and simplified end-to-side anastomoses. ASME, J.Biomechanical Engineering, Vol. 121(3), 1999, pp. 265-72.

31. M. Moubachir, J.P. Zolésio. Optimal control of fluid-structure interaction systems: the case of a rigid solid. INRIA, Rapport de Recherche, No 4611, 2002.

32. T. P. O'Brien, T. McGloughlin. Computational Fluid Dynamics Analysis of Blood Flow in compliant arterial grafts. $12^{\text {th }}$ Conference of the European Society of Biomechanics, Dublin, 2000.

33. K. Perktold, M. Hofer, G. Karner, W. Trubel, H. Schima. Computer Simulation of Vascular Fluid Dynamics and Mass Transport: Optimal Design of Arterial Bypass Anastomoses. Proceedings of ECCOMAS 98, pp. 484-489, K. Papailion and others Editors, John Wiley and Sons, Ltd, 1998.

34. O. Pironneau. Optimal Shape Design for Elliptic Systems. Springer-Verlag, Springer Series in Computational Physics, New York, 1984.

35. A. Quarteroni, M. Tuveri, A. Veneziani. Computational Vascular Fluid Dynamics: Problems, Models and Methods. Computing and Visualization in Science, Vol. 2 (2000), pp. 163-197.

36. A. Quarteroni, L. Formaggia. Mathematical Modelling and Numerical Simulation of the Cardiovascular System in Modelling of Living Systems, Handbook of Numerical Analysis Series (P.G. Ciarlet e J.L. Lions Eds), Elsevier, Amsterdam, 2003.

37. A. Quarteroni, A. Valli. Numerical Approximation of Partial Differential Equations. Springer-Verlag, Berlin, 1994.

38. A. Quarteroni, R. Sacco, F. Saleri. Numerical Mathematics. Springer, New York, 2000.

39. T. Slawig. Domain Optimization for the stationary Stokes and Navier-Stokes Equations by an embedding domain Tecnique. Ph.D. Thesis, Tu-Berlin, 1998.

40. D.A. Steinman, B. Vinh, C.R. Ethier, M. Ojha, R.S Cobbold, K.W Johnston. A numerical simulation of flow in a two-dimensional end-to-side anastomosis model. Journal of Biomechanical Engineering, Vol. 115 (1), 1993, pp. 112-118.

41. C.A. Taylor, C.P. Cheng, L.A. Espinosa, B.T. Tang, D. Parker, R.J. Herfkens. In Vivo Quantification of Blood Flow and Wall Shear Stress in the Human Abdominal Aorta during lower limb exercise. Annals of Biomedical Engineering, Vol. 30, 2002, pp. $402-08$.

42. A. Veneziani Mathematical and Numerical Modelling of Blood Flow Problems. Ph.D. Thesis, Politecnico di Milano, 1998.

43. D. Wells, J.P. Archie, C. Kleinstreuer The Effect of Carotid Artery Geometry on the Magnitude and Distribution of Wall Shear Stress Gradients. Journal of Vascular 
Surgery, Vol. 23, 1996, pp. 667-678.

44. S.S. White, C.K. Zarins, D.P. Giddens, H.S. Bassiouny, F. Loth, A. Jones, S.Glagov. Haemodynamic patterns in two flow models of end-to-side anastomoses: effect of pulsatility, flow division, Reynolds number and hood length. Journal of Biomechanical Engineering, Vol. 115, pp. 104-111, Feb. 1993.

45. K. Yosida. Functional Analysis. Springer-Verlag, Berlin, 1974.

46. C. Zarins, D. Giddens, B. Bharadvaj, V. Sottiurai, R. Mabon, S. Glagov. Carotid Bifurcation Atherosclerosis: quantitative correlation of plaque localization with flow velocity profiles and wall shear stress. Circ. Res. 53, pp. 502-514, 1983. 